\title{
Incidence of Diabetes and its Control Measures in AIDS Patients
}

\section{Hima Bindu $\mathrm{A}^{1 *}$ and Soumya $\mathrm{D}^{2}$}

${ }^{1}$ Department of Biotechnology, Andhra Vidyalaya P.G College, Osmania University, Hyderabad, India

${ }^{2}$ Department of Microbiology, Chaitanya Degree and P.G College, Kakatiya University, Warangal, India

\begin{abstract}
HIV and diabetes are both chronic diseases that significantly affect the human lifestyle and their quality of living. When these two diseases coexist, their treatment would be overwhelming for patients. Highly active antiretroviral therapy (HAART) became the standard of care in HIV infection. As the treatment for HIV has been developed and the access to therapy has been improved, the incidence of HIV-associated diabetes began to grow. Diabetes can be an important consequence of HAART. This review describes various metabolic complications such as AIDS-related insulin resistance, Glucose Intolerance and Hyperglycemia after HAART and also gives a brief account on the control measures.
\end{abstract}

Keywords: Acquired immunodeficiency syndrome; Human Immunodeficiency Virus; Antiretroviral therapy; highly active antiretroviral therapy; Combination antiretroviral therapy; Diabetes Mellitus; Hypoglycemia; Insulin Resistance; Glucose Intolerance; Hyperglycemia

Abbreviations: AIDS: Acquired Immunodeficiency Syndrome; HIV: Human Immunodeficiency Virus; ART: Antiretroviral Therapy; HAART: Highly Active Anti-Retroviral Therapy; cART: Combination Anti-Retroviral Therapy; NRTIs: Nucleotide Reverse Transcriptase Inhibitors; NNRTIs: Non-Nucleotide Reverse Transcriptase Inhibitors; PIs: Protease Inhibitors; DM: Diabetes Mellitus; MetS: Metabolic Syndrome; CVD: Cardiovascular disease; IGT: Impaired Glucose Tolerance; OADs: Oral Anti Diabetic Drugs

\section{Introduction}

Acquired immunodeficiency syndrome (AIDS) is a caused by the human immunodeficiency virus (HIV) that belongs to a subset of retroviruses called lentiviruses [1]. HIV/AIDS is the fourth leading cause of mortality and remains the greatest public health crisis in today's world [2]. The main reason is not only a major public health issue, but also a socio-economic and developmental crisis that affects all sectors of the population [3]. Continuous efforts are being made to identify novel effective therapies that can assist in controlling the spread of HIV [1].

Chronic human immunodeficiency virus (HIV) infection is characterized by defects in the immune system including depletion of $\mathrm{CD}^{4+} \mathrm{T}$-cells and impaired T-cell function. The immune suppression in AIDS patients is the major cause of various opportunistic diseases [4]. HIV/AIDS patients are prone to the development of not only opportunistic infections [5] such as various bacterial, fungal, protozoal and viral diseases, but also endocrine disorders such as diabetes mellitus (DM) [6].

Human immunodeficiency virus and host cell protein interaction network has provided an opportunity for development of novel antiviral therapeutics targeted to host proteins required for virus infection [7]. Successful antiretroviral therapy (ART) suppresses viral replication [8]. The introduction of the highly active antiretroviral therapy (HAART) also called as combination antiretroviral therapy (cART) in 1996 has drastically reduced the morbidity and mortality associated with the HIV infection. The potent combination of antiretroviral (ARV) therapy has changed the course of HIV disease from an invariably fatal illness to a chronic but manageable one [9]. There are currently 20 antiretroviral drugs that have been approved for the treatment of HIV. They were divided into six classes of ART which inhibit HIV replication [10]. Each of these classes of drugs inhibits HIV replication at different stages in HIV life cycle. The decision of ART depends upon the $\mathrm{CD}^{4+}$ count of each individual [11].

Highly active antiretroviral therapy (HAART) became the standard of care in HIV infection [12] and includes the combination of three different types of highly effective anti-HIV-1 drugs, including nucleotide reverse transcriptase inhibitors (NRTIs), non-nucleotide reverse transcriptase inhibitors (NNRTIs) and non-peptidic viral protease inhibitors (PIs) $[1,13]$. This therapy helped in substantially reducing viral load, slowing HIV replication, increasing $\mathrm{CD}^{4}$ lymphocyte numbers, and reducing the incidence of opportunistic infections [14].

NRTIs are currently an essential part of highly active antiretroviral therapy (HAART) for the treatment of HIV. However, the use of some dideoxynucleotide analogues may be limited by mitochondrial toxicity [15]. NNRTIs based antiretroviral therapy (ART) regimens have been recommended and widely used because of their reliable efficacy, low pill burden, and low cost [16]. The PIs have come into use as the human immunodeficiency virus (HIV) encodes within its genome an aspartyl protease that is required to process viral precursor polyproteins and is essential for the proper formation of infectious HIV virions.

With the advent of antiretroviral therapy (ART), the incidence of perinatal HIV-1 transmission has decreased from 20-25\% to less than $2 \%$. However, recent studies suggest that exposure to antiretroviral medications may have marked adverse effects, independent of HIV status [17]. Adverse events are common in patients receiving ARV therapy [18]. Current highly active antiretroviral therapy (HAART) for the treatment of HIV infection is associated with long term side effects

*Corresponding authors: Hima Bindu A, P.G Department of Biotechnology, Andhra Vidyalaya P.G College, Affiliated to Osmania University, Hyderabad, India; E- Mail: bindu2889@gmail.com

Received November 10, 2011; Accepted December 11, 2011; Published December 16, 2011

Citation: Hima Bindu A, Soumya D (2011) Incidence of Diabetes and its Control Measures in AIDS Patients. J Diabetes Metab 2:169. doi:10.4172/21556156.1000169

Copyright: (c) 2011 Hima Bindu A, et al. This is an open-access article distributed under the terms of the Creative Commons Attribution License, which permits unrestricted use, distribution, and reproduction in any medium, provided the original author and source are credited. 
[15]. The effect of HAART can be assessed by classifying the individuals into three groups - (1) HIV seronegative, (2) HIV infected not using HAART, and (3) HIV infected using HAART [19].

Highly active antiretroviral therapy (HAART) is generally associated with disturbance of lipid metabolism, fat mass distribution and insulin resistance [20]. HIV-related opportunistic infectious diseases and autoimmune disorders have also been observed [21]. It has also been found out that protease inhibitor use is associated with metabolic abnormalities [22]. Patients who receive protease inhibitors (PIs)-based antiretroviral therapy (ART) show a higher Hepatitis C viremia than those treated with nonnucleoside reverse transcriptase inhibitors (NNRTI) [23]. Even with combination antiretroviral therapy (cART), the durability of HIV control is limited by many factors such as adherence to treatment, drug toxicity, bioavailability etc [24].

\section{HIV Treatment - Diabetes}

HIV and diabetes are both chronic diseases that significantly affect the human lifestyle and their quality of living. When these two diseases coexist, their treatment would be overwhelming for patients. HIV disease being an important global problem, increasing prevalence of Diabetes Mellitus (DM) is observed among these patients [25]. HIV infection is characterized by an immunodeficient state caused by active replication of the virus. In order to achieve maximum suppression of viral replication, the use of combination therapy served as an important consideration [14].

Glucose is the prime fuel as the source of energy for humans. However, the glucose levels in circulation have to be very tightly maintained. Alterations in the normal glucose levels in blood will lead to abnormal physiological states causing either hypoglycemia (low glucose levels) or hyperglycemia (high glucose levels) [26]. Diabetes mellitus is a disease of abnormal glucose metabolism resulting in hyperglycemia due to either a deficiency of insulin secretion or insulin resistance or both [27]. Diabetes is one of the most frequent metabolic diseases and is widely distributed in various populations; its prevalence appears to be increasing rapidly [28]. People with diabetes have problems converting food in to energy. It is also defined as chronic disorders of carbohydrate metabolism due to the lack of insulin result in the hyperglycemia [29]. The current estimated prevalence of diabetes worldwide is 285 million people [30].

The prevalence of diabetes mellitus is rising at an alarming rate. Epidemiological studies of diabetes mellitus have shown that gender, age, and ethnic background are important factors when considering the development of diabetes mellitus and its complications [31]. Various other factors like metabolic syndrome (MetS) [32], Obesity have shown a profound effect. Increased obesity in the population has lead to increased number of diabetic patients [33]. Depending on the etiology of the DM, factors contributing to hyperglycemia include reduced insulin secretion, decreased glucose utilization, and increased glucose production [34]. Classic signs and symptoms of diabetes include polyuria, polydipsia, polyphagia, weight loss, headache, tachycardia, palpitations, and blurred vision. The primary pathophysiological defects include excessive hepatic glucose production, impaired peripheral glucose uptake by insulin-sensitive tissues, and insufficient insulin secretion/increased $\beta$-cell apoptosis [35].

The prevalence of diabetic complications was high [36]. Type 2 diabetes mellitus is a chronic disease [37] with severe late complications and high mortality [38]. Diabetes mellitus (DM) long- term complications are progressive and almost resulting by chronic exposure to high blood levels of glucose resulting from defects in insulin metabolism and dysfunction in carbohydrate, lipid and protein metabolism [39]. It is also associated with long-term complications of peripheral nervous system and the central nervous system [40], has a profound effect on the skeleton and can lead to osteoporosis [41]. Cardiovascular disease (CVD) is a major complication and a leading cause of early death among persons with diabetes [42]. Diabetes affects patients' physical, emotional and psychological status, leading to many diseases and complications which burden patients, family and community [43].

The risk factors for diabetes, such as family history of the disease, obesity, and sedentary lifestyle, are important in people with HIV [44] The traditional risk factors for DM are responsible for most of the increased risk in the HIV infected population [45]. As the treatment for HIV has been developed and the access to therapy has been improved, the incidence of HIV-associated diabetes began to grow. The incidence of new-onset diabetes in HIV-infected persons was found to be significantly high. Three subgroups of patients with diabetes and HIV have been identified

\section{- Patients with preexisting diabetes who contract HIV}

- Those who are diagnosed to have diabetes at onset of HIV infection

\section{- Those who develop hyperglycemia after start of therapy}

The recent development of highly active antiretroviral therapy (HAART) has drastically improved the life expectancy of AIDS patients but the long-term use of novel, potent antiviral agents has lead to new problems and complications [46]. Increasing numbers of AIDS patients are receiving protease inhibitors (PIs) for the treatment of their HIV infection [47]. The prevalence of AIDS-related insulin resistance, Glucose Intolerance and Diabetes increased dramatically after HAART [25].

\section{Insulin resistance}

Insulin is needed to help control the amount of sugar in the body. Insulin is one of the most extensively studied proteins in many fields [48]. Insulin resistance is a condition in which the body cannot use insulin effectively and higher concentrations of insulin are required to exert normal effects. Fasting insulin levels may be high because of compensatory insulin secretion by the pancreas [49]. As a result, blood sugar and fat levels rise. High glucose levels can be a side effect of HIV drugs. Specifically, the protease inhibitors (PIs) can make it difficult for insulin to get glucose into the cells. This is called insulin resistance. It can lead to pre-diabetes and diabetes. Insulin resistance may result from antiviral medication directly impairing glucose uptake in the muscle. Insulin resistance may also increase the risk of coronary heart disease [50].

When people are insulin resistant, their muscle, fat, and liver cells do not respond properly to insulin. As a result, their bodies need more insulin to help glucose enter cells. The pancreas tries to keep up with this increased demand for insulin by producing more. Eventually, the pancreas fails to keep up with the body's need for insulin. Excess glucose builds up in the bloodstream, setting the stage for diabetes. Many people with insulin resistance have high levels of both glucose and insulin circulating in their blood at the same time [51]. Insulin Resistance occurs as a result of disturbances in lipid metabolism and increased levels of circulating fatty acids that accumulate within the insulin sensitive muscle, liver and adipose tissues [52]. 
Reports of insulin resistance and the development of overt diabetes increased with the routine clinical use of PIs [14]. The various mechanisms that contribute to insulin resistance include chronic inflammatory changes caused by HIV, and side effects of antiretroviral medication, such as the interference of protease inhibitors (PIs) in the activity of glucose transporters, the damage to mitochondria caused by NRTIs etc [44].

Insulin resistance among treated $\mathrm{HIV}$-infected patients is multifactorial. It includes common contributors to insulin resistance such as obesity, genetic influences, and physical inactivity; antiretroviral drugs and lipodystrophy [53]. The coexistence of obesity, glucose intolerance, dyslipidemia, and hypertension, is termed as insulin resistance syndrome [54]. Insulin resistance is manifested early in the natural history of the disease but glucose tolerance remains normal because of a compensatory increase in insulin secretion and hyperinsulinemia [55].

\section{Glucose intolerance}

Impaired glucose tolerance (IGT) is a pre-diabetic state of dysglycemia that is associated with insulin resistance and increased risk of cardiovascular pathology [56]. Impaired glucose tolerance and diabetes mellitus can occur in HIV patients receiving HAART [57]. Protease inhibitors do not increase risk of glucose intolerance or insulin resistance among pregnant women [58].

\section{Hyperglycemia}

Diabetes is a chronic disorder characterized by high levels of glucose in the blood and is a common disorder affecting individuals of all ages [59]. Hyperglycemia is a symptom of Diabetes [60]. Increased risk of diabetes mellitus is observed in people taking HAART, specifically protease inhibitors (PIs). Hyperglycemia, new-onset diabetes mellitus, exacerbation of existing diabetes mellitus, and diabetic ketoacidosis has been reported in HIV-infected patients taking PIs. Diabetes mellitus, one of the most prevalent diseases in developing world, is a metabolic disorder characterized by hyperglycemia and other metabolic alterations due to relative or absolute insulin deficiency [61]. Treatment with HIV protease inhibitors (PIs) and infection with hepatitis $\mathrm{C}$ virus increase the risk of hyperglycemia and diabetes in people with HIV. The risk of developing hyperglycemia is the same with all PIs. People who are older, obese [62], family history with diabetes are also at greater risk for developing hyperglycemia [60]. Hyperglycemia is also associated with excessive free radical generation and oxidant stress [63].

\section{Control Measures}

Current therapies require lifelong treatment which can be associated with significant toxicity and economic cost. In some instances, the use of cART may be restricted by contraindications, drug resistance, or limited access. There is a need for simple treatment options which provide sustained potency, limited toxicity, and a high genetic barrier to development of resistance [64]. Patients with HIV should be screened for diabetes at diagnosis, at onset of HAART therapy, and three to six months after HAART [65].

Understanding the glucose disturbances that are possible with PI therapy, performing appropriate screening for glucose intolerance and diabetes and making prudent changes in HIV therapy when necessary, and treating patients for alterations in glucose metabolism are the key components of care for at-risk patients [14]. Maintaining a healthy weight and increasing physical activity, Diabetes treatment, with oral agents or insulin may be useful [44]. Counseling will be an important requirement for Anti Retroviral treatment [3]. Research suggests that physical activity is inversely related to numerous metabolic disorders in people who are living with HIV [66].

Despite all the advances in diabetes treatment, education remains the cornerstone of diabetes management. Diabetes education is important in improving diabetes self-management and providing effective diabetes treatment. Differences in diet, exercise levels, stress and other factors may all affect blood glucose levels, so people should be educated about how these factors affect them and the various control measures [67].

Psychosocial support is an integral part of effective diabetes management; it is of utmost importance in patients who have to handle the double stress of diabetes and HIV. Diet, physical activity/ exercise and cessation of smoking are the important measures. Oral Anti Diabetic Drugs (OADs) should be used in patients with Diabetes and HIV with great care. Insulin is the drug of choice for management of diabetes with HIV. HIV-infected patients should be taught how to dispose of lancets, glucose strips, insulin syringes, pens and needles, to prevent HIV transmission [65].

PI-based regimes should be avoided in patients at high risk of developing diabetes. Patients should be counselled about the potential risks, discomforts and benefits of HAART, and encouraged to follow a healthy lifestyle while monitoring glycemia regularly. The effective management of diabetes in HIV infected patients requires a thorough understanding of pathophysiology and pharmacology [65].

\section{Conclusion}

The recent development of highly active antiretroviral therapy (HAART) has drastically improved the life expectancy of AIDS patients but the long-term use of novel, potent antiviral agents has lead to new problems and complications. Current therapies require lifelong treatment which can be associated with significant toxicity and economic cost. Hence there is a need for simple treatment options which provide sustained potency, limited toxicity, and a high genetic barrier to development of resistance. The effective management of diabetes in HIV infected patients is therefore required to avoid undesirable consequences.

\section{Acknowledgements}

I would like to express my fullest gratitude to all my friends who helped me in successful completion of this review article.

\section{References}

1. Pande PP (2009) Computational Approach towards designing potential HIV inhibitors. J Antivir Antiretrovir 1: 082-085.

2. Guha P, Sardar P (2011) Prevalence of Paediatric HIV Infection in Eastern India-First report. J AIDS Clinic Res 2:127.

3. Nsimba SED, Irunde H, Comoro C (2010) Barriers to ARV Adherence among HIVIAIDS Positive Persons taking Anti-Retroviral Therapy in Two Tanzanian Regions 8-12 Months after Program Initiation. J AIDS Clinic Res 1:111.

4. Bhardwaj A, Parikh R, Daoko J, Singh L, Shamoon FE, et al. (2009) Cardiovascular Manifestation of HIV: Review. J Antivir Antiretrovir 1: 011-016.

5. HIV-related opportunistic diseases: UNAIDS Technical Update (1998)

6. Kabati CIA, Maurice HB, Mselle T, Magdalena Urio (2010) Evaluation of the Prevalence of Insulin Dependent Diabetes Mellitus in HIV/AIDS Patients in Muhimbili National Hospital. TaJONAS 1: 165-173.

7. Neurath AR, Lackman-Smith C (2009) Prevention of Human Immunodeficiency 
Citation: Hima Bindu A, Soumya D (2011) Incidence of Diabetes and its Control Measures in AIDS Patients. J Diabetes Metab 2:169. doi:10.4172/21556156.1000169

Page 4 of 5

Virus Type 1 Transmission by Pharmaceuticals Targeted to Host Proteins Required for Virus Infection? Consideration of Farnesyl Thiosalicylic Acid, a Ras Inhibitor. J Antivir Antiretrovir 1: 072-075.

8. Tan DBA, Yong YK, Tan HY, French M, Kamarulzaman A, et al. (2010) Characteristics of Natural Killer Cells in Malaysian HIV Patients Presenting with Immune Restoration Disease After ART. J AIDS Clinic Res 1:102.

9. Reginald OO, Mohammed MH, Olayinka AT, Sani GB, Tobi-Ajayi E, et al. (2011) Hematological and Metabolic Toxicities of Current Antiretroviral Regimens in Ahmadu Bello University Teaching Hospital Shika Zaria, Northern Nigeria. J AIDS Clinic Res S2:002.

10. Naga Anusha P (2011) Antiretroviral Strategies for Treatment of HIV. J Antivir Antiretrovir R1.

11. Amin J, De Lazzari E, Emery S, Martin A, Martinez E, et al. (2010) Simplifi cation with Fixed-Dose Tenofovir-Emtricitabine or Abacavir-Lamivudine in Treatment Experienced, Virologically Suppressed Adults with Hiv Infection Combined Analysis of Two Randomised, Non-Inferiority Trials Bicombo and Steal. J AIDS Clinic Res 1:103.

12. Pineda JA, Alcamí J, Blanco JR, Blanco J, Boix V, et al. (2011) Hot Immunological Topics in HIV Infection. J AIDS Clinic Res 2:118.

13. Kino T, Mirani M, Alesci S, Chrousos GP (2003) AIDS-related lipodystrophy/ insulin resistance syndrome. Horm Metab Res 35: 129-136.

14. Spollett GR (2006) Hyperglycemia in HIVIAIDS. Diabetes Spectr 19: 163-166.

15. Herzmann C, Smith C, Johnson MA, Byrne P, Terenghi G, et al. (2010) A Prospective, Double Blind, Randomised, Placebo Controlled Trial Evaluating Acetyl-L-Carnitine (ALCAR) for the Prevention of Distal Symmetric Polyneuropathy in HIV Infected Individuals. J AIDS Clinic Res 1:108.

16. Soe AN, Tansuphasawadikul S, Phonrat B, Boonpok L, Tepsupa S, et al (2010) Early Viral Suppression Predicting Long-term Treatment Success Among HIV Patients Commencing NNRTI-based Antiretroviral Therapy. J Antivir Antiretrovir 2: 033-037.

17. Míguez MJ, Rosenberg R, Burbano X, Malow R (2011) Cholesterol as a Mediator of Alcohol-Induced Risks for Respiratory Disease Hospitalizations among People Living With HIV. J AIDS Clinic Res S1:001.

18. Moore RD, Kumar R (2010) Outcomes of Ritonavir-Boosted Protease Inhibitor versus Non-Nucleoside Reverse Transcriptase Regimens in a Clinical Practice Cohort. J Antivir Antiretrovir 1: 013-019.

19. Brown TT, Cole SR, Li X, Kingsley LA, Palella FJ et al. (2005) Antiretroviral therapy and the prevalence and incidence of diabetes mellitus in the multicente AIDS cohort study. Arch Intern Med 165: 1179-1184.

20. Gontran M, Jerome LT, Yvonne AM, Mavoungou-Poaty V, Elie M, et al. (2009) Effects of IM28 on HIV-1 and Metabolic Disorders-induced Highly Active Antiretroviral Therapy in Gabonese Patients. J Antivir Antiretrovir 1: 076-081.

21. Corrêa RB, Schmidt FR, Silva MLCF, Costa FHR, Rosso AL, et al. (2010) Holmes' Tremor in an HIV Positive Patient Worsened by Immune Recovery Infl ammatory Syndrome (IRIS). J AIDS Clinic Res 1:105

22. Murata H, Hruz PW, Mueckler M (2000) The mechanism of insulin resistance caused by HIV protease inhibitor therapy. J Biol Chem 275: 20251-20254.

23. Mata RC, Mira JA, Rivero A, López-Cortés LF, Torres-Tortosa M, et al. (2010) Nevirapine-based Antiretroviral Therapy is Associated with Lower Plasma Hepatitis C Virus Viral Load among HIV/Hepatitis C Virus-Coinfected Patients. J AIDS Clinic Res 1:110.

24. Meynard JL, Morand-Joubert L, Chêne G, Landman R, Pinta A, et al. (2011) Two-Year Observational Study in Patients Infected with Drug-Resistant HIV-1 and Treated with the Fusion Inhibitor Enfuvirtide: The ZOOM Cohort. J AIDS Clinic Res 2:114.

25. Wiwanitkit V (2007) Primary care for diabetes in HIV-infected patients. OJHAS 6: $1-5$

26. Uppu RM, Parinandi NL (2011) Insulin Sensitization and Resistance Interrelationship Revisited with a Quantitative Molecular Model Approach. J Diabetes Metab 2:106e.

27. Sai YRKM, Dattatreya A, Anand SY, SureshBabu D, Sandeep Heni RS (2011) Biomarkers of Internal Origin and Their Significance in Diabetes and Diabetic Complications. J Diabetes Metab R1:001.

28. Belmokhtar F, Belmokhtar R, Dali-Sahi M, Charef M (2011) Risk Factors
Associated With Type 2 Diabetes Mellitus in West Region of Algeria, Maghnia. J Diabetes Metab 2:148

29. Ramanathan K, Karthick H, Arun N (2010) Structure Based Drug Designing for Diabetes Mellitus. J Proteomics Bioinform 3: 310-313.

30. Joffe B, Distiller L, Landau S, Blacking L, Klisiewicz A (2010) Spectrum of Autoimmune Disorders in Type 1 Diabetes - A Cross-Sectional Clinical Audit. J Diabetes Metab 1:112.

31. Li YW, Aronow WS (2011) Diabetes Mellitus and Cardiovascular Disease. $J$ Clinic Experiment Cardiol 2:114.

32. Kaneko M, Suzuki H, Watanabe H, Oda $E$, Aizawa $Y$ (2011) Metabolic Syndrome is a Poor Predictor of Incident Diabetes Compared with Hemoglobin A1c (Hba1c) in a General Japanese Population. J Diabetes Metab S:2.

33. Nichol A, Chandra Sekar M (2011) Successful Management of Extremely Insulin-Resistant Obese Diabetic Patient with Insulin Glargine, U-500 Regular Insulin and Pramlintide. J Diabetes Metab 2:143.

34. Shanker JH, Mahmood SE, Joshi MC, Shaifali I (2011) Obesity Indices amongs Diabetics in an Urban Population of Western Nepal. J Diabetes Metab 2:134.

35. Fouqueray P, Leverve X, Fontaine E, Baquié M, Wollheim C, et al. (2011) Imeglimin - A New Oral Anti-Diabetic that Targets the Three Key Defects of type 2 Diabetes. J Diabetes Metab 2:126.

36. Abougalambou SSI, Hassali MA, Sulaiman SAS, Abougalambou AS (2011) Prevalence of Vascular Complications among Type 2 Diabetes Mellitus Outpatients at Teaching Hospital in Malaysia. J Diabetes Metab 2:115.

37. Mungrue K, Roper LA, Chung T (2011) Assessment of Weight Loss in the Management of Patients with Type 2 Diabetes Mellitus in Primary Care in Trinidad. J Diabetes Metab 2:120.

38. Samadi N, Safavi M, Mahmoodi M (2011) Impact of Quality of Life Education on Self-Concept among Type 2 Diabetes Patients. J Diabetes Metab 2:132.

39. da Silva SB, Costa JP, Pintado ME, Ferreira DC, Sarmento B (2010) Antioxidants in the Prevention and Treatment of Diabetic Retinopathy - A Review. J Diabetes Metab 1:111.

40. Chen D, Huang H, Xing Y, Liu Y, Xu Y, et al. (2011) A New Vanadium Complex Improves the Spatial Learning and Memory by Activation of Caveolin-MAPKCREB Pathway in Diabetic Mice. J Diabetes Metab 2:114.

41. Vestergaard $P$ (2011) Diabetes and Bone. J Diabetes Metab S: 1.

42. Huffman FG, Vaccaro JA, Nusrath NS, Zarini GG (2011) The Effect of Carbohydrate Amount, Quality and Type on Arterial Pulse Pressure in CubanAmericans with and Without Type 2 Diabetes. J Nutr Food Sci 1:106.

43. Ali ZH (2011) Health and Knowledge Progress among Diabetic Patients afte Implementation of a Nursing Care Program Based on Their Profile. J Diabetes Metab 2:121.

44. Monroe A (2009) HIVIAIDS and diabetes: minimizing risk, optimizing care BETA 21: 38-44.

45. Butt AA, McGinnis K, Rodriguez-Barradas MC, Crystal S, Simberkoff M (2009) HIV infection and the risk of diabetes mellitus. AIDS 23: 1227-1234.

46. Kino T, Chrousos GP (2003) AIDS-related insulin resistance and lipodystrophy syndrome. Curr Drug Targets Immune Endocr Metabol Disord 3: 111-117.

47. Salehian B, Bilas J, Bazargan M, Abbasian M (2005) Prevalence and incidence of diabetes in HIV-infected minority patients on protease inhibitors. J Natl Med Assoc 97: 1088-1092.

48. Ramachandra S (2011) Do we need yet another Insulin? J Diabet Metabo 2:0e4

49. http://www.aids-ed.org/aidsetc?page $=c g-603$ insulin_resistance

50. Grinspoon S (2003) Mechanisms and strategies for insulin resistance in acquired immune deficiency syndrome. Clin Infect Dis 2: S85-90.

51. http://diabetes.niddk.nih.gov/dm/pubs/insulinresistance/

52. Ragheb R, Medhat AM (2011) Mechanisms of Fatty Acid-Induced Insulin Resistance in Muscle and Liver. J Diabetes Metab 2:127.

53. De Wit S, Sabin CA, Weber R, Worm SW, Reiss P (2008) Incidence and risk factors for new-onset diabetes in HIV-infected patients: the Data Collection on Adverse Events of Anti-HIV Drugs (D:A:D) study. Diabetes Care 31: 1224 1229 
Citation: Hima Bindu A, Soumya D (2011) Incidence of Diabetes and its Control Measures in AIDS Patients. J Diabetes Metab 2:169. doi:10.4172/21556156.1000169

54. Misra A, Vikram NK (2004) Insulin resistance syndrome (metabolic syndrome) and obesity in Asian Indians: evidence and implications. Nutrition 20: 482-491.

55. Florez H, Scranton R, Farwell WR, DeFronzo RA, Ezrokhi M, et al. (2011) Randomized Clinical Trial Assessing the Efficacy and Safety of BromocriptineQR when Added to Ongoing Thiazolidinedione Therapy in Patients with Type 2 Diabetes Mellitus. J Diabetes Metab 2:142.

56. http://en.wikipedia.org/wiki/Impaired_glucose_tolerance

57. http://www.medscape.com/viewarticle/410384_3

58. Hitti J, Andersen J, McComsey G, Liu T, Melvin A et al. (2003) Protease inhibitor-based antiretroviral therapy and glucose tolerance in pregnancy: AIDS Clinical Trials Group A5084. Am J Obstet Gynecol 196: e1-7.

59. Ribeiro C, de Alencar Mota CS, Voltarelli FA, de Araújo MB, Botezelli JD, et al. (2010) Effects of Moderate Intensity Physical Training in Neonatal AlloxanAdministered Rats. J Diabetes Metab 1:107.

60. Fact Sheets: Side Effects of Anti-HIV Medications-Health Information for Patients (2005) AIDS info.

61. Kumar R, Kumar AN, Ahmed S (2011) Changes in Erythrocyte Membrane in Type-2 Diabetes Mellitus with and without Dyslipidemia. J Diabetes Metab 2:141.
62. Kablan A, Saunders RA, Szkudlarek-Mikho M, Chin JB, Bosio RM, et al. (2010) Prieurianin Causes Weight Loss in Diet-Induced Obese Mice and Inhibits Adipogenesis in Cultured Preadipocytes. J Diabetes Metab 1:101.

63. El Asrar MA, Adly AAM, El Hadidi E, Gharib M (2011) Serum and Urinary Nitrites and Nitrates and Doppler Sonography in Detection of Early Diabetic Complications. J Diabetes Metab 2:117.

64. Pinola M, Lazzarin A, Antinori A, Carosi G, Di Perri G, et al. (2010) Lopinavir/ ritonavir + tenofovir Dual Therapy versus Lopinavir/ritonavir-Based Triple Therapy in HIV-Infected Antiretroviral Naïve Subjects: The Kalead Study. J Antivir Antiretrovir 2: 056-062.

65. Kalra S, Kalra B, Agrawal N, Unnikrishnan A (2011) Understanding diabetes in patients with HIVIAIDS. Diabetol Metab Syndr 3: 2.

66. Santos-Lozano A, Garatachea N (2011) Physical Activity Measurements Using Accelerometers and Pedometers in HIV-Infected People. J AIDS Clinic Res 2:126

67. David SK, Upadhayaya N, Siddiqui MK, Usmani AM (2010) Knowledge Discovery Technique for Web-Based Diabetes Educational System. J Health Med Informat 1:102. 